\title{
ON THE PIN-AND-SLOT DEVICE OF THE ANTIKYTHERA MECHANISM, WITH A NEW APPLICATION TO THE SUPERIOR PLANETS*
}

\author{
CHRISTIÁN C. CARMAN, Universidad Nacional de Quilmes/CONICET, \\ ALAN THORNDIKE, University of Puget Sound, and \\ JAMES EVANS, University of Puget Sound
}

Perhaps the most striking and surprising feature of the Antikythera mechanism uncovered by recent research is the pin-and-slot device for producing the lunar inequality. ${ }^{1}$ This clever device, completely unattested in the ancient astronomical literature, produces a back-and-forth oscillation that is superimposed on a steady progress in longitude - nonuniform circular motion. ${ }^{2}$ Remarkably, the resulting motion is equivalent in angle (but not in spatial motion in depth) to the standard deferent-plus-epicycle lunar theory. Freeth et al. gave a proof of this equivalence, which is, however, a very complicated proof. ${ }^{3}$ One goal of the present paper is to offer a simpler proof that would have been well within the methods of the ancient astronomers and that, moreover, makes clearer the precise relation of the pin-andslot model to the standard epicycle-plus-concentric and eccentric-circle theories.

On the surviving portions of the Antikythera mechanism, only two devices are used to account for inequalities of motion. The first is the pin and slot, used for the lunar inequality. And the second is the nonuniform division of the zodiac scale, which, we have argued, was used to model the solar inequality. ${ }^{4}$ The second would obviously be of no use in representing planetary inequalities; so a natural question is to ask whether the pin-and-slot mechanism could be modified and extended to the planets, especially to the superior planets, for which the likely mechanical representations are less obvious than they are for the inferior planets. The second goal of this paper, then, is to examine the relation of the pin-and-slot model to the standard concentric-plusepicycle theory for the retrograde motion of the planets. We shall see that, indeed, the pin and slot can be applied to the planets and that this device again gives an exact representation of the motion in angle, though not in depth.

Scholars have pondered the various holes and structures on the main solar gear b1, and wondered whether these might represent the remnants of a lost part of the mechanism designed for reproducing planetary motion. ${ }^{5}$ The evidence is frustratingly sparse. An inscription on the front cover of the mechanism appears to describe planetary phenomena, including "stations", and another inscription on the back cover mentions Venus by name, ${ }^{6}$ but the only hardware surviving is a single wheel of 63 teeth $(\mathrm{r} 1)$ which has no function in the published reconstruction of the solar and

\footnotetext{
* This paper is partly based on data processed, with permission, from the archive of experimental investigations by the Antikythera Mechanism Research Project (Freeth et al. (ref. 1)) in collaboration with the National Archaeological Museum of Athens.
} 
lunar portions of the mechanism. It is not much to go on. That a planetary display could be possible has been shown by Michael Wright, who, in a feat of mechanical virtuosity, constructed one that works and that is based on a full representation of deferent-and-epicycle theory. ${ }^{7}$ We ourselves published a more modest proposal, in which the mechanism would have offered a display of the key events in the planets' synodic cycles, but not a full-on kinematic display showing the progress of the planets around the zodiac with retrograde motion. ${ }^{8}$ However, in our view, the realization that the pin-and-slot mechanism could be applied to the planets (when we know that it is in fact used for the Moon) suggests the possibility of a simpler kinematic model. We remain agnostic about whether the Antikythera mechanism offered a full-on kinematic display, or a more modest display of information about planetary phases, or perhaps a display of the mean motions. However, like Hipparchos, we feel it is worth the attention of geometers to investigate the explanation of the same phenomena by means of hypotheses that are so different. ${ }^{9}$

So the third goal of this paper is to present a new approach for the planetary display in the Antikythera mechanism. We will follow the idea proposed by Michael Wright and others, according to which the Antikythera mechanism displayed planetary longitudes on the front dial, using pointers concentric with those of the Moon and Sun and sharing the same zodiac scale. But we will use a pin-and-slot mechanism to produce the inequality with respect to the Sun (retrograde motion). A remarkably simple reconstruction of the planetary display becomes possible, which also fits comfortably onto the four-spoke main solar gear.

\section{The Main Characteristics of the Device for the Lunar Inequality}

The device for producing the lunar inequality consists of four gears called e5, e6, $\mathrm{k} 1$, and k2, illustrated in Figure 1 (left). ${ }^{10}$ On the Antikythera mechanism these are all of equal tooth number - 50 - but the key requirement is that they be equal by pairs: $\mathrm{k} 1$ and $\mathrm{e} 5$ are equal; and $\mathrm{k} 2$ and e 6 are equal. The input motion is from an axle (actually, a hollow pipe) at $\mathrm{E}$ that turns e5 at the rate of the Moon's mean sidereal frequency; following Freeth et al. we designate this frequency $\omega_{\text {si }}$. Concentric with e5, but turning freely from it, is a large wheel e3, which turns at the rate of the Moon's line of apsides. From a modern point of view, the orientation of the Moon's major axis does not stay invariable. Rather, the Moon's elliptical orbit itself turns in its own plane, so that the perigee advances in the same direction as the Moon moves, taking about 9 years to go all the way around the zodiac. The ancient astronomers were aware that the position of fastest speed in the Moon's orbit itself advances around the zodiac, the Greeks modelling the motion geometrically and the Babylonians by means of arithmetical period relations. In the Antikythera mechanism the advance of the Moon's perigee is modelled by letting e 3 turn with a frequency $\omega_{\mathrm{n}}$ (again using the notation of Freeth et al.).

Riding on e 3 at centre $\mathrm{C}_{1}$ is gear $\mathrm{k} 1$, which is driven by e5 and therefore also turns with respect to absolute space at frequency $\omega_{\mathrm{si}}$. Gear $\mathrm{k} 2$ turns about an axis, $\mathrm{C}_{2}$, also 
attached to e 3 but slightly offset from $\mathrm{C}_{1}$. The offset is achieved by using a stepped stud, with its larger diameter centred at $\mathrm{C}_{1}$ and its smaller diameter centred at $\mathrm{C}_{2}$, as shown in the right side of Figure 1 and in the inset perspective view. Thus k1 turns about $\mathrm{C}_{1}$ and $\mathrm{k} 2$ turns about $\mathrm{C}_{2}$. Wheel $\mathrm{k} 1$ has a small pin, which engages a radial slot in $\mathrm{k} 2$. Thus $\mathrm{k} 1$, turning at a uniform speed, drives $\mathrm{k} 2$, producing a quasi-sinusoidal oscillation in the speed of $\mathrm{k} 2$ - a little faster when the pin is closer to $\mathrm{C} 2$ and a little slower when farther from $\mathrm{C} 2$ - which is superimposed on the mean motion. The motion of $\mathrm{k} 2$ is transferred to e6, which rotates independently of both e3 and e5 about axis E, and communicates the nonuniform motion of the Moon to the other parts of the mechanism. Uniform motion in (at e5) is transformed into non-uniform motion out (at e6) around the same axis. ${ }^{11}$

\section{Simple Proof for the Function of the Lunar Pin-and-Slot Mechanism}

We shall now offer a simple demonstration of the efficacy of this device, and will also make clear its relation to the ordinary tools of Greek theoretical astronomy, namely epicycles and eccentrics. Let us for the moment imagine that the Moon's line of apsides does not revolve, but remains in the same spatial orientation. We

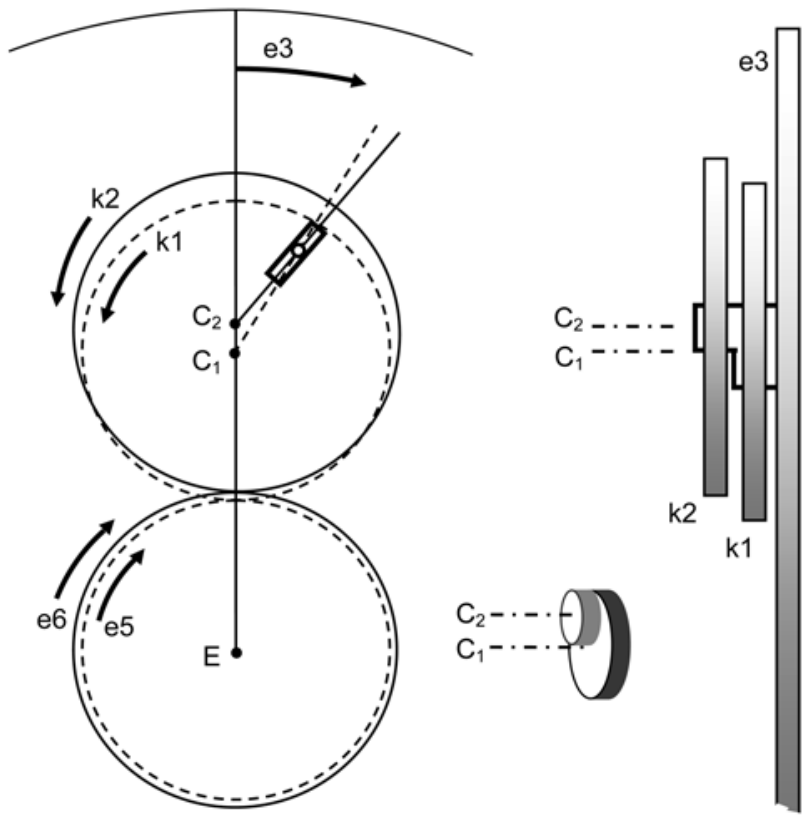

FIG. 1. The device for producing the inequality in the Moon's motion around the zodiac, as reconstructed in ref. 1. The edge-on view (at right) shows how the stepped stud achieves different axes of rotation for $\mathrm{k} 1$ and $\mathrm{k} 2$. 
shall construct a representation of the standard Hipparchian epicyclic lunar theory, and superimpose on it a diagram of the pin-and-slot device from the Antikythera mechanism.

Consider first the epicycle theory. Refer to Figure 2. Circle k1 now represents the Moon's deferent circle, with centre at the Earth $\mathrm{C}_{1}$. The epicycle's centre D is carried around clockwise (eastward) at the rate of the Moon's mean motion. Thus angle $\theta$ increases uniformly with time. Simultaneously, the Moon M moves counterclockwise on its epicycle, but at exactly the same rate, so angle $\alpha$ remains equal to $\theta$. Angle $\alpha$, the Moon's epicyclic anomaly, increases uniformly at the angular frequency $\omega_{\mathrm{a}}$. The direction of the Moon as seen from the Earth is line $\mathrm{C}_{1} \mathrm{M}$. Thus, at the moment shown in the diagram, the angular position of the Moon beyond its point of fastest motion is $\theta+q$ (where $q$ is called the lunar equation).

Now suppose that Figure 2 represents the model realized in the Antikythera mechanism. In Figure 2, we are in the frame of reference of wheel e3. Alternatively, one can think of the motion of the line of apsides as being suppressed for the time being. Wheel $\mathrm{k} 1$ turns uniformly about centre $\mathrm{C}_{1}$; mounted on $\mathrm{k} 1$ is a pin at $\mathrm{D}$. (We may think of $\mathrm{k} 1$ as being somewhat larger than shown, so that the pin need not be exactly at the perimeter of the wheel.) Wheel k2 rotates about a distinct centre $\mathrm{C}_{2}$ (slightly off-centre from $\mathrm{C}_{1}$ ). $\mathrm{k} 2$ has a slot (represented by the heavy dashed line), which points in the direction of $\mathrm{C}_{2}$.

Imagine that $\mathrm{k} 1$ was originally oriented so that pin $\mathrm{D}$ lay in the direction of fastest motion (in the direction of line $\mathrm{C}_{1} \mathrm{C}_{2}$ ). But $\mathrm{k} 1$ has now turned far enough that $\mathrm{D}$ lies at angle $\theta$ as viewed from $\mathrm{C}_{1}$. D will be seen from $\mathrm{C}_{2}$ at a different and larger angle $\phi$. The question is: just what is the size of this angle $\phi$ ? Because of the equality of $\theta$ and $\alpha$, it follows that DM remains parallel to $\mathrm{C}_{2} \mathrm{C}_{1}$. Then, if we chose the eccentricity $\mathrm{C}_{1} \mathrm{C}_{2}$ in the pin-and-slot mechanism to be equal to the radius DM in the epicycle theory, $\mathrm{C}_{2} \mathrm{DMC}_{1}$ must form a changing parallelogram with $\phi$ always equal to $\theta+q$. Thus, the motion in angle in the pin and slot mechanism agrees precisely with the motion in angle of the epicycle theory. That is, a point, such as $\mathrm{Z}$, on the rim of $\mathrm{k} 2$ moves about $\mathrm{C}_{2}$ in a perfect circle, but at variable angular speed. The angular position of $\mathrm{Z}$ observed from $\mathrm{C}_{2}$ is the same as the angular position of $\mathrm{M}$ as seen from $\mathrm{C}_{1}$. Now, we may also allow wheel e 3 to move, carrying line $\mathrm{C}_{1} \mathrm{C}_{2}$ around with it, and the demonstration remains intact. Moreover, the rotating ensemble now models the lunar inequality in detail, with its rotating line of apsides.

\section{Relation to the Eccentric-Circle Theory}

As is well known (and was equally well known in Antiquity) the epicycle-plusconcentric model is geometrically equivalent to an eccentric-circle theory. See Figure 3. Since DM remains parallel to $\mathrm{C}_{2} \mathrm{C}_{1}$, it follows that the physical motion of $\mathrm{M}$ through space, resulting from the dual motion of $\mathrm{D}$ around the deferent $\mathrm{k} 1$ and of $\mathrm{M}$ around the epicycle, is uniform motion on a perfect circle, shown in dashed line, which is centred at $\mathrm{C}_{3}$, one epicycle radius below $\mathrm{C}_{1}$. In the typical proof of the 


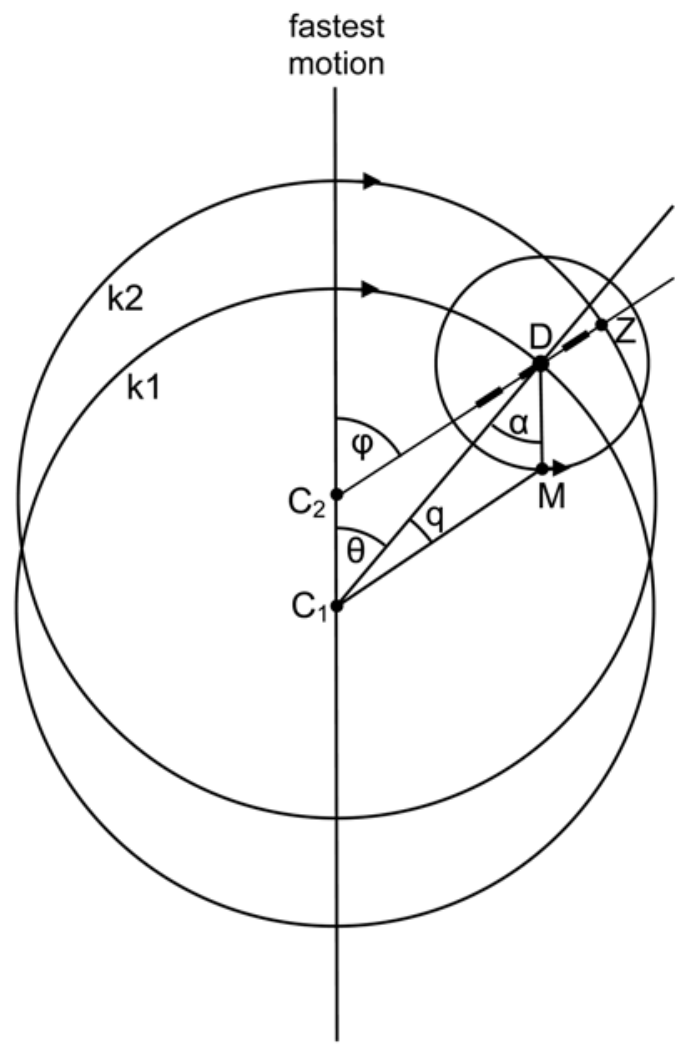

FIG. 2. Pin-and-slot mechanism for the lunar inequality in the Antikythera mechanism, compared with the epicycle-plus-concentric theory in the standard astronomy of Hipparchos or Ptolemy.

equivalence of eccentric and epicycle, one focuses on parallelogram $\mathrm{C}_{1} \mathrm{C}_{3} \mathrm{MD}$. (But it is parallelogram $\mathrm{C}_{2} \mathrm{C}_{1} \mathrm{MD}$ that embodies the quasi-equivalence of the pin-and-slot mechanism to the epicycle theory.)

Suppose $\mathrm{C}_{1}$ represents the Earth. Then, as the ancient astronomers knew, there were two equivalent theories: the epicycle-plus-concentric (epicycle DM plus circle $\mathrm{k} 1$ ) and the eccentric-circle theory (circle k3 with no epicycle). The proof of this equivalence was given by Ptolemy in the Almagest, and earlier by Theon of Smyrna, who cited Adrastos as his authority. Traditionally, based on some remarks of Ptolemy, the proof of the equivalence of these two theories is ascribed to Apollonios of Perge. But no one really knows when it was first proven. ${ }^{12}$

As far as is known to us, there is no extant ancient mention of the quasi-equivalence of the pin-and-slot mechanism to the epicycle theory. This is a quasi-equivalence because the pin and slot model produces the same motion in angle, but not the same physical motion in space as the epicycle model. The output of the pin and slot 


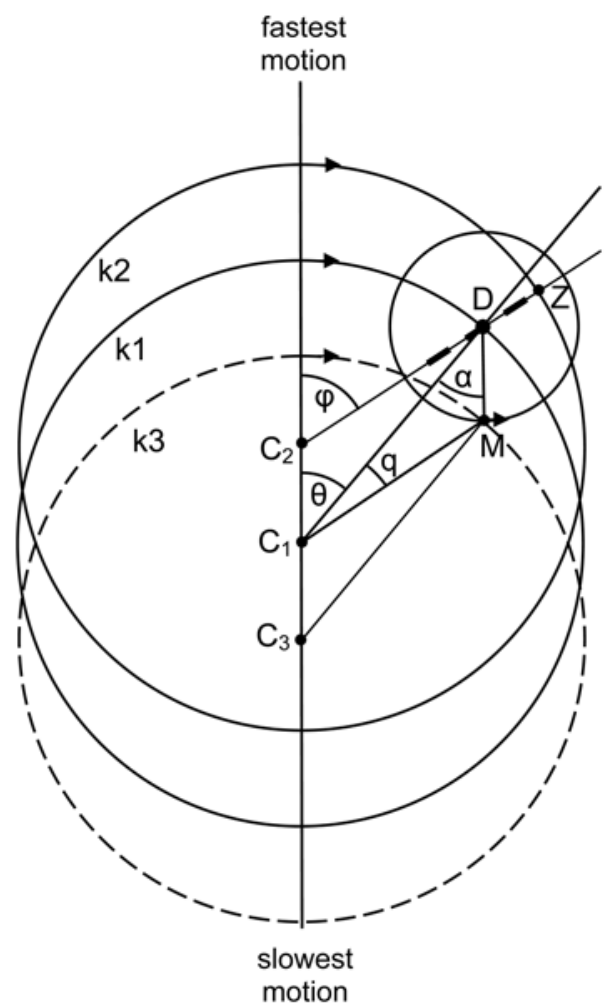

FIG. 3. Three models compared: pin-and-slot (wheels k1 and k2), epicycle-plus-concentric (epicycle DM and circle k1), and eccentric circle (circle k3).

model is a point moving at non-uniform speed on a circle $\mathrm{k} 2$. But the output of the epicycle-plus-concentric model (or of the eccentric-circle model) is a point moving uniformly around circle $\mathrm{k} 3$.

One must suppose that an ancient Greek astronomer trained in the philosophicalgeometrical tradition of Hipparchos and Ptolemy would not have regarded the pin-and-slot mechanism as a "realistic" representation of the lunar theory, for the pin-and-slot mechanism suppresses the motion "in depth", but nevertheless gives an account of the motion in longitude that agrees with what the epicycle theory would prescribe. Aristotle, too, would have considered it not an appropriate solution, as he insists that each simple body (e.g., a celestial orb) should be animated by a single simple motion. And here, the final output motion is the rotation of e6, which consists in a steady rotation with a superimposed oscillation.

Did the ancient mechanic who designed the Antikythera mechanism realize the equivalence in angle of the pin and slot mechanism to the epicycle theory? If so, how is it that no proof of the equivalence survives? Or was this mechanism considered a 
rough-and-ready approximation to epicycle motion — good for giving the final output angle, but not necessarily considered exact? In a way, the striking contrast between applied mechanics and accepted celestial physics should not surprise us. There is a well-documented example of a similar contrast. Greek astronomers grounded in the philosophical-geometrical tradition (for example, Theon of Smyrna, early second century A.D.) wrote treatises on deferent and epicycle theory while their contemporaries were busy mastering and adapting the non-geometrical planetary theory of the Babylonians. ${ }^{13}$ The philosophically-based astronomy of the high road explicitly endorsed uniform circular motion as the only motion proper to celestial bodies while the numerically-minded astronomers (who needed quick and reasonably reliable results for the purposes of astrology) made free and easy with nonuniformity of motion. In a similar way, it is possible that mechanical tricks of the trade such as the pin-and-slot mechanism were used in a craft tradition of model-building, quite apart from the practices of the "serious" (i.e., geometrically-minded) astronomers. In the medieval tradition of equatoria, one also finds representations of planetary theory that are not "realistic". ${ }^{14}$ On the other hand, Figures 2 and 3 show that a proof of the equivalence in angle would have been well within the reaches of contemporary Greek geometry.

\section{Choice of Active and Passive Elements}

An interesting feature of the pin-and-slot geometry is that the equation of centre function changes its form if the roles of the pin and slot are reversed. See Figure 4 (left), which shows the pin and slot as found on the mechanism. $\mathrm{k} 1$ turns about $\mathrm{C}_{1}$ with the uniform, mean motion. It is easy to show that the equation $q$ of centre is given by

$$
\sin q=\frac{e \sin \theta}{\sqrt{1+e^{2}-2 e \cos \theta}},
$$

where $e=b / r$. Here $b=\mathrm{C}_{1} \mathrm{C}_{2}$ is the distance between the axes and $r=\mathrm{C}_{1} \mathrm{D}$ is the fixed distance of the pin from the centre $\mathrm{C}_{1}$ of wheel $\mathrm{k} 1$. This is the ordinary expression for the equation of centre in standard Ptolemaic solar theory.

But if (right side of Figure 4) the pin is instead placed in k2, and the slot is cut into $\mathrm{k} 1$ and directed toward $\mathrm{C}_{1}$, then we find instead

$$
\sin q=e \sin \theta
$$

where again $e=b / r$, but now $r$ is the fixed distance of the pin from $\mathrm{C}_{2}$. The more complex expression has become a simple sinusoidal oscillation. ${ }^{15}$ So we are again left to wonder: did the mechanic have a deep understanding of the equivalence theorem, or is it just chance that the pin is placed in $\mathrm{k} 1$ rather than in $\mathrm{k} 2$ ? Perhaps, as $\mathrm{k} 1$ drives $\mathrm{k} 2$, he would have thought of the pin as the active element and therefore chosen to place it on $\mathrm{k} 1$, the active wheel. 


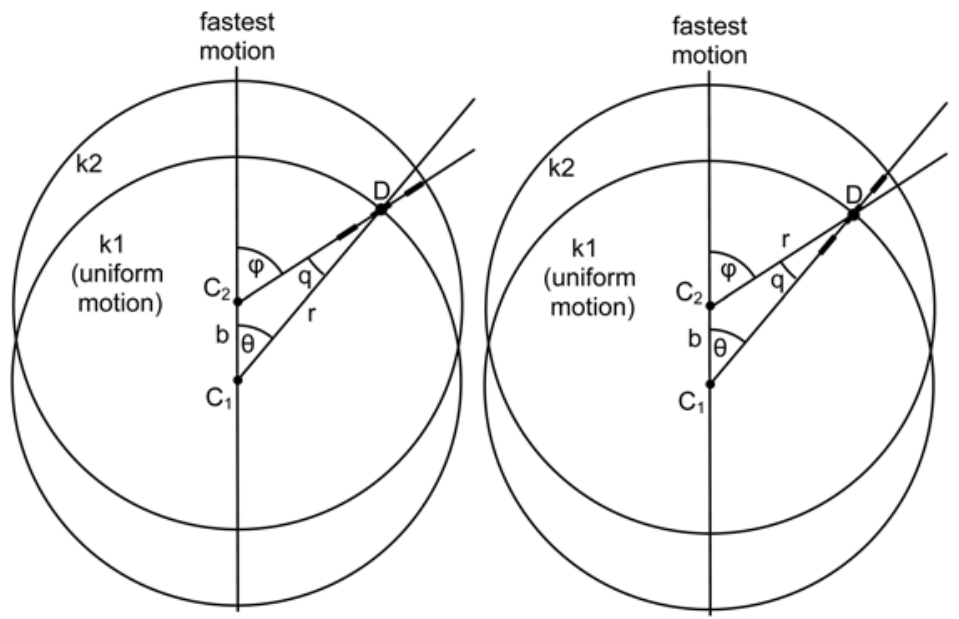

FIG. 4. The effect of reversing the roles of the pin and slot.

\section{Transfer of the Lunar Pin-and-Slot Mechanism to the Case of the Outer Planets}

In their discussion of the lunar pin-and-slot mechanism, Freeth et al. say:

As discussed above, in absolute terms, e5 rotates at the rate of $\omega_{\mathrm{si}}$ and e 3 rotates at the rate of $\omega_{\mathrm{n}}$. So, relative to e3, e5 rotates at the rate of $\omega_{\mathrm{si}}-\omega_{\mathrm{n}}=\omega_{\mathrm{a}}$. Since e 5 and k1 both have 50 teeth, relative to e3, k1 rotates at the rate of $\omega_{\mathrm{a}}$. This is the critical factor that ensures that the anomaly introduced by the pin-and-slot mechanism has the period of the anomalistic month, as required by Hipparchos' lunar theory. ${ }^{16}$

Now, we want to transfer the basic idea of the lunar inequality to the planets. The lunar inequality is an inequality with respect to the zodiac - or, if we want to be more exact and take into account the fact that the line of apsides slowly advances, an inequality with respect to wheel e3. This is why the wheels that produce the inequality ( $\mathrm{k} 1$ and $\mathrm{k} 2$ ) must ride on wheel $\mathrm{e} 3$. But retrograde motion is an inequality with respect to the Sun. ${ }^{17}$ So, in order to represent an epicycle and deferent model of the planets we need:

(a) a gear rotating with the sidereal period, i.e., the period of the mean longitude (in the Moon model, e5).

(b) a "big" carrier gear rotating at $\omega_{\mathrm{n}}=\omega_{\mathrm{si}}-\omega_{\mathrm{a}}$, i.e., at the difference between the rates of mean longitude and of the epicyclic anomaly. In the case of the Moon, the epicyclic anomaly increases in the opposite direction to the sidereal motion, so, in the case of a planet, where both the epicycle and the deferent rotate in the same direction, the "big" gear should rotate at $\omega_{\mathrm{si}}+\omega_{\mathrm{a}}$. (It is not clear that the outset whether 
the difference in the directions of motion on the epicycle will cause problems or not. We shall see that all will be well.)

(c) the set of other gears, with the correct eccentricity, for the pin and slot.

Now, for an outer planet, the sum of the planet's sidereal frequency and its anomalistic frequency is equal to the frequency of the Sun's mean motion: $\omega_{\mathrm{si}}+\omega_{\mathrm{a}}=\omega_{\odot}$. (This familiar result follows from the construction in Figure $5 .{ }^{18}$ ) This means that, for all the outer planets, the "big" gear (corresponding to e 3 in the lunar mechanism) should rotate at the same period, and the period is that of the mean motion of the Sun. Fortunately, we do have a big gear rotating at this period, the solar gear, b1. And, fortunately also, this gear is rotating about the correct axis, i.e., the axis of the pointers. Therefore, we need only place on each of three spokes of b1 the pin-andslot mechanism of a superior planet. This coincidence seems to increase significantly the plausibility of this proposal for, besides the theoretical reason (to have the same style of mechanism for the planets and the Moon), we have an empirical reason, i.e., we actually have a big gear rotating at the rate we need.

So, for each outer planet, we only need to have a gear, concentric with b1, rotating with the sidereal frequency $\omega_{\text {si }}$ for this planet. Then, the addition of a pin-and-slot mechanism rotating at frequency $\omega_{\odot}$ (so, riding on b1), with the correct eccentricity, would produce the apparent motion in angle of the planet.

\section{Pin-and-Slot Compared to Apollonios's Planetary Epicycle}

In the preceding section we gave a plausibility argument that the pin-and-slot mechanism can effectively mimic planetary motion. We shall show now that a pin-and-slot mechanism will account in detail for the motion in angle of the planet. Consider the motion in longitude of a superior planet according to standard epicycle theory, as shown in Figure 5. $\mathrm{C}_{1}$ is the Earth. Imagine that at one moment the centre D of the epicycle and the planet $\mathrm{P}$ both lay on the vertical straight line in the direction of fastest motion. But at the moment represented in the figure, a certain amount of time $t$ has elapsed, and the planet's mean longitude has increased by $\theta=\omega_{\mathrm{si}} t$. The epicyclic anomaly has increased by $\alpha=\omega_{\mathrm{a}} t$. The longitude of the Sun has increased by $\odot=\omega t$. Because of the standard constraint linking the motion of the Sun and the superior planet we have $\odot=\theta+\alpha$, and $\omega_{\odot}=\omega_{\text {si }}+\omega_{\text {a }}$ At this moment, the direction from the Earth to the planet is in the line $\mathrm{C}_{1} \mathrm{P}$. The planet's angular distance from the direction of fastest motion is $\theta+q$, where $q$ is the equation of the epicycle. $\theta$ increases steadily with time, while $q$ undergoes an oscillation.

Now let us consider matters as seen in the frame of reference of the revolving solar gear b1. Here the situation is shown in Figure 6. The line from $\mathrm{C}_{1}$ to the Sun points in a fixed direction (vertical in the figure). Thus, we have rotated Figure 6 counterclockwise by the angle $\odot$ (compared with Figure 5). The angular position of the planet with respect to the vertical reference line (i.e., the elongation of the planet from the Sun) is 


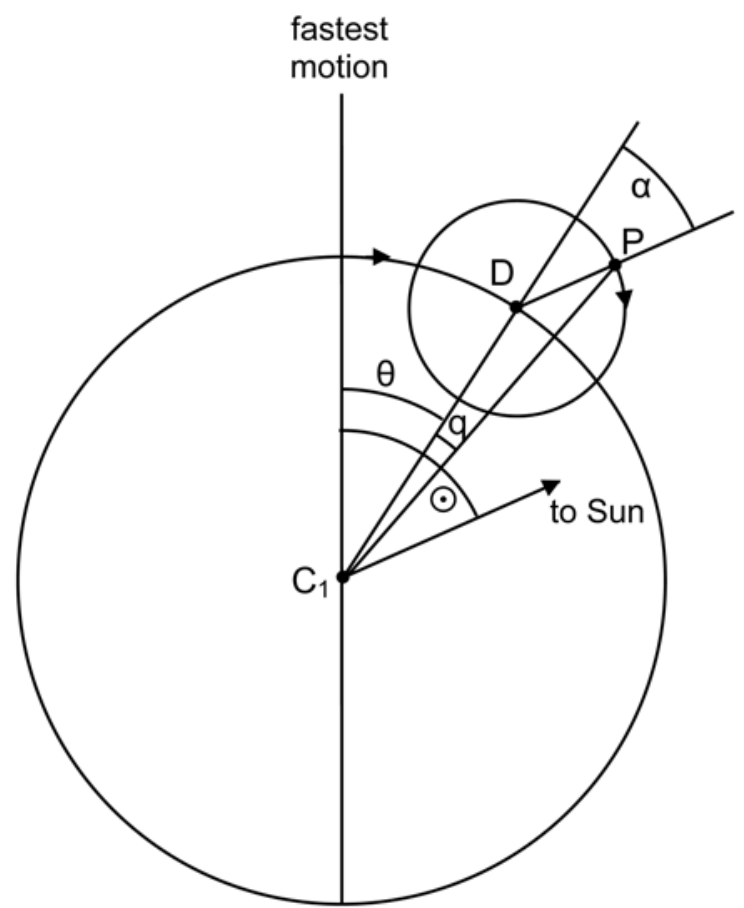

FIG. 5. Epicycle theory for the motion of a superior planet. The rotating line from the epicycle's centre D to the planet P must stay parallel to the rotating line from the Earth $\mathrm{C}_{1}$ to the Sun. So $\theta+\alpha=\odot$.

$$
\eta=\theta+q-\odot .
$$

Note that $\eta$ increases to the left as time goes by. That is, in the frame of gear b1, D moves counterclockwise. This is simply because $\omega_{\text {si }}<\omega_{\odot}$. Note that Figure 6 is a sort of mirror image of Figure 2. In Figure 2, we had D moving clockwise around the deferent, while $\mathrm{M}$ went counterclockwise on the epicycle. Here, with the planetary theory, as viewed in the frame of reference of b1, we have just the opposite: D moves counterclockwise, while P moves clockwise on the epicycle. Thus, in spite of the fact the planets move in the opposite direction on their epicycles than does the Moon, the conditions necessary for the theorem do apply.

Because the direction DP of the planet in the epicycle remains parallel to the direction from the Earth to the Sun, we have DP always parallel to $\mathrm{C}_{2} \mathrm{C}_{1}$. If we choose a circle $\mathrm{k} 2$, with centre $\mathrm{C}_{2}$ located below $\mathrm{C}_{1}$ by a distance equal to $\mathrm{DP}$, it follows that the quadrilateral $\mathrm{C}_{1} \mathrm{PDC}_{2}$ is a changing parallelogram; thus $\mathrm{C}_{2} \mathrm{D}$ is always parallel to $\mathrm{C}_{1} \mathrm{P}$. Now suppose that $\mathrm{D}$ represents a pin in a wheel $\mathrm{k} 1$, driving wheel $\mathrm{k} 2$ by means of a radial slot. (In Figure 6, the slot is suggested by the heavy dashed line, and one should imagine $\mathrm{k} 2$ as larger.) Then a fixed point $\mathrm{Z}$ on $\mathrm{k} 2$ in the direction of $\mathrm{C}_{2} \mathrm{D}$ will move so that $\mathrm{C}_{2} \mathrm{Z}$ is always parallel to the direction $\mathrm{C}_{1} \mathrm{P}$. Again, this is an equivalence in angle only. To get back into the frame of the fixed stars, we just rotate Figure 6 


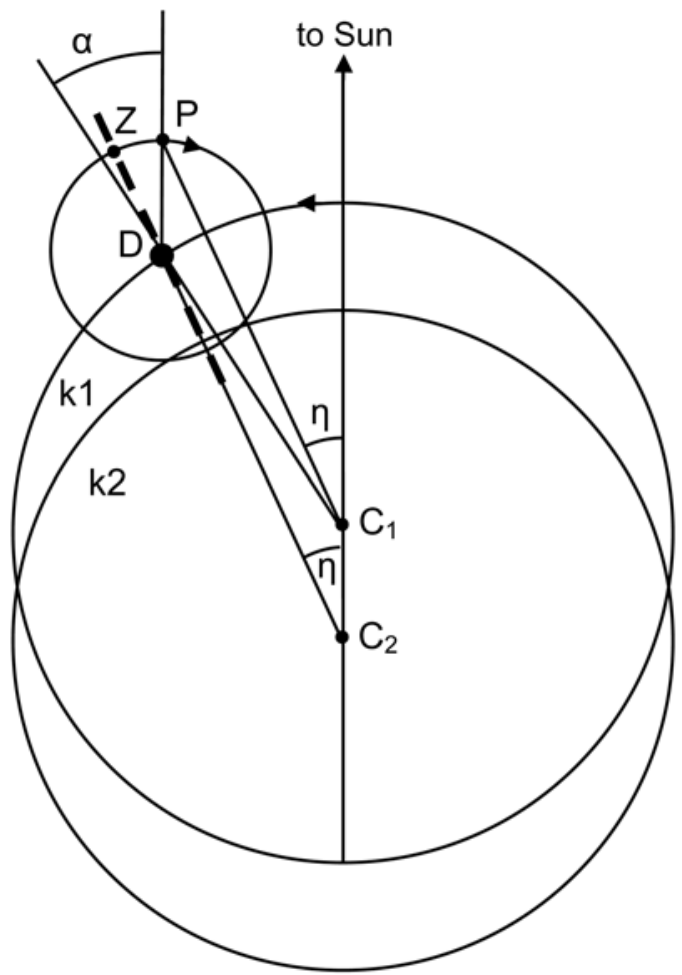

FIG. 6. The epicyclic theory of a superior planet (viewed in the frame of reference of the Sun wheel), superimposed on a pin-and-slot mechanism.

clockwise by angle $\odot$; but the parallelness of $\mathrm{C}_{2} \mathrm{D}$ and $\mathrm{C}_{1} \mathrm{P}$ will not be disturbed.

This works because of a remarkable circumstance. In the case of the lunar theory, the theorem will only hold if the Moon moves backwards on the epicycle. (A theory with both $\mathrm{D}$ and $\mathrm{M}$ moving clockwise, even if $\mathrm{M}$ completes its motion on the epicycle in one anomalistic period, will not satisfy the conditions of Figure 2. In this case, a pin and slot might still give a reasonable approximation to the epicycle theory, but it would not be an exact angular match.)

In the case of the planets, the direction of $\mathrm{P}$ on the epicycle is clockwise - in the same direction as D. So it might be thought that the theorem could not possibly apply. But the situation is saved by the fact that the angular frequency of the Sun is greater than that of the superior planets. So, when we rotate Figure 5 counterclockwise, to get into the frame of reference of the solar wheel, we end up with Figure 6 - the planet moving clockwise on the epicycle, but the epicycle moving counterclockwise on the deferent. The opposite directions rescue the theorem.

A date for the design of the Antikythera mechanism can be placed between the late third century B.C. and the early first century B.C. It seems a very open question 
whether the mechanic understood the deep theoretical complexities of the pin-andslot mechanism; if he did, we might reasonably suspect that the mechanism comes from rather later in the possible range, after a thorough understanding of planetary theories had been arrived at. But if the mechanic used the pin and slot as a sort of ad-hoc way of imposing an oscillation on a steady forward motion, he need not have been aware of the existence of an equivalence proof. In this case, he was perhaps "lucky" — though his mechanical instincts were still remarkable.

The Antikythera mechanism contributes significant new evidence for the debate over realism and instrumentalism in ancient planetary theory. To put it crudely, the mechanic was a happy instrumentalist. The lunar pin-and-slot mechanism represents the motion of the Moon satisfactorily in angle, but renounces a description of the motion in space. The planetary pin-and-slot mechanism (if it existed!) represents another such goal-oriented bit of modelling. And of course, the use of a non-uniformly divided zodiac to model the solar anomaly fits right into this pattern. There is an irony here, in that Ptolemy, when he gives directions for obtaining the longitude of a planet from his theories, essentially gives a procedure for eliminating any consideration of the depth in space. And he was hardly an instrumentalist. But the final output of a realistic theory may look like the output of an instrumentalist's black box.

\section{Obtaining the Mean Sidereal Motion of an Outer Planet}

We have a working pin and slot that effectively model the motion in angle in Apollonian planetary theory. The next requirement for a display of the zodiacal motion of a superior planet is a gear turning about the b axis at the rate of the planet's sidereal frequency $\omega_{\text {si }}$. One simple way to achieve the sidereal rotation is to use a stationary gear, concentric with the $\mathrm{b}$ axis, fixed to a plate behind the dial plate. Refer to Figure 7A. Gear $\mathrm{u}$ with $u$ teeth is fastened to the back of the plate. Gears $\mathrm{x}$ and $\mathrm{y}$ are carried on an axle that rides around on $\mathrm{b} 1$ in a period equal to the year. $\mathrm{x}$ and $\mathrm{y}$ are rigidly fixed to one another (as suggested by the dark line with diamonds that connects them in the figure). Finally, y engages with a gear $z$ that is concentric with the $b$ axis, but is free to turn independently. The frequency of gear b1 with respect to fixed space is $\omega_{\odot}=1$ revolution/year. b1 rotates clockwise as viewed from above the plate in the diagram, and $\omega_{\odot}$ is considered positive. Presently, we shall work out the frequency $\omega_{\mathrm{z}}$ of gear $\mathrm{z}$ with respect to absolute space and show that it can be made equal to $\omega_{\mathrm{si}}$.

An alternative way to achieve the same result, shown in Figure 7B, is to make use of the stationary squared-off boss, visible in the centre of Figure 8. It is possible that this boss was fixed with respect to the plate behind $\mathrm{b} 1$, and its squared-off portion may have served as a gear seat. ${ }^{19}$ Figures $7 \mathrm{~A}$ and $7 \mathrm{~B}$ are entirely equivalent in terms of generating the output frequency $\omega_{\mathrm{z}}$. In each case we are working with a stationary gear $\mathrm{u}$ and the rotating solar gear $\mathrm{b} 1$ that turns with a period of a year. The arrangement of 7B inverts the vertical space order of gears $\mathrm{x}$ and $\mathrm{y}$.

Let us now work out the rates of rotation. Refer to Figure 9. (Figure 9 and the argument that follows apply equally well to the models of Figure 7A and Figure 7B: 


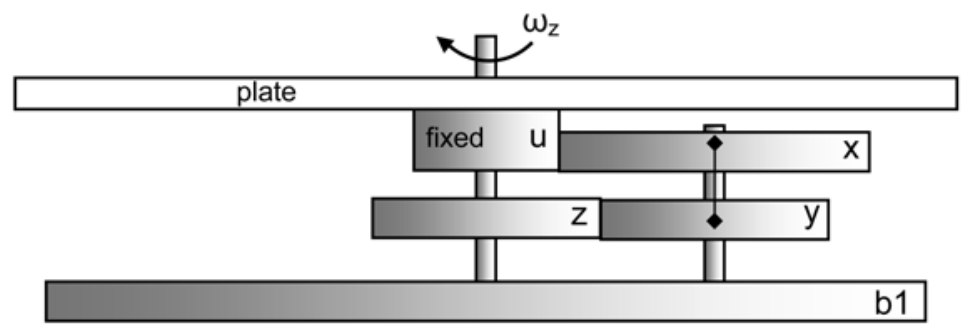

FIG. 7A. One way to get wheel z rotating at the mean sidereal frequency of a superior planet. The example is for Mars, with $u, x, y, z=37,79,58$ and 58 teeth.

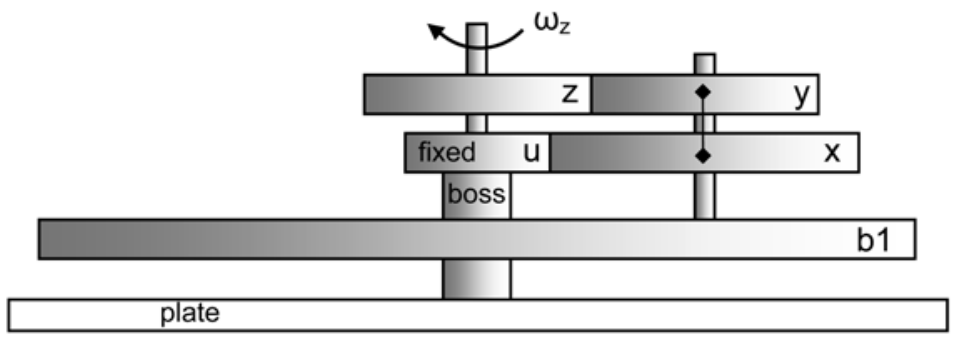

FIG. 7B. A second way to get wheel $\mathrm{z}$ rotating at the mean sidereal frequency of a superior planet, using a stationary boss.

it does not matter whether $\mathrm{u}$ and $\mathrm{x}$ are above or below $\mathrm{y}$ and $\mathrm{z}$.) The top part of Figure 9 shows the positions of wheels $\mathrm{u}$ and $\mathrm{x}$ before (left) and after (right) a rotation of the main Sun wheel $\mathrm{b} 1$ has taken place. Before the motion begins, point $\mathrm{p}$ on wheel $\mathrm{x}$ is oriented as shown. The main solar wheel, carrying the axle of $x$, rotates clockwise through $\theta$; the situation is now as in the upper right. Point $\mathrm{p}$ has been carried to a new position. The number $n$ of teeth that have been engaged on $\mathrm{u}$ is given by

$$
n=u(\theta / 360) \text {. }
$$

The same number of teeth must have been engaged on wheel $\mathrm{x}$, so it follows that

$$
\begin{gathered}
\phi=360(n / x) \\
=\theta u / x .
\end{gathered}
$$

The angle in absolute space through which wheel $\mathrm{x}$ has rotated is $\theta+\phi=\theta(1+u / x)$.

Now the lower part of Figure 9 examines the fates of wheels y and z. Before the rotation (left) the initial position of point $\mathrm{q}$ on wheel $\mathrm{z}$ is shown. After the rotation (right side of figure), $\mathrm{z}$ will have rotated through an angle $\psi$ in absolute space, so $\mathrm{q}$ will be in a new position. Depending on the tooth numbers $u, x, y, z$, it can be the case that $\mathrm{q}$ has rotated either clockwise or counterclockwise. We want $\mathrm{q}$ to have moved clockwise, corresponding to the sidereal motion of the planet, so in the diagram we have placed $\mathrm{q}$ below the horizontal. Because $\mathrm{x}$ and $\mathrm{y}$ are rigidly fastened together, the angles marked $\phi$ in the upper right and lower right are the same. The number $m$ 


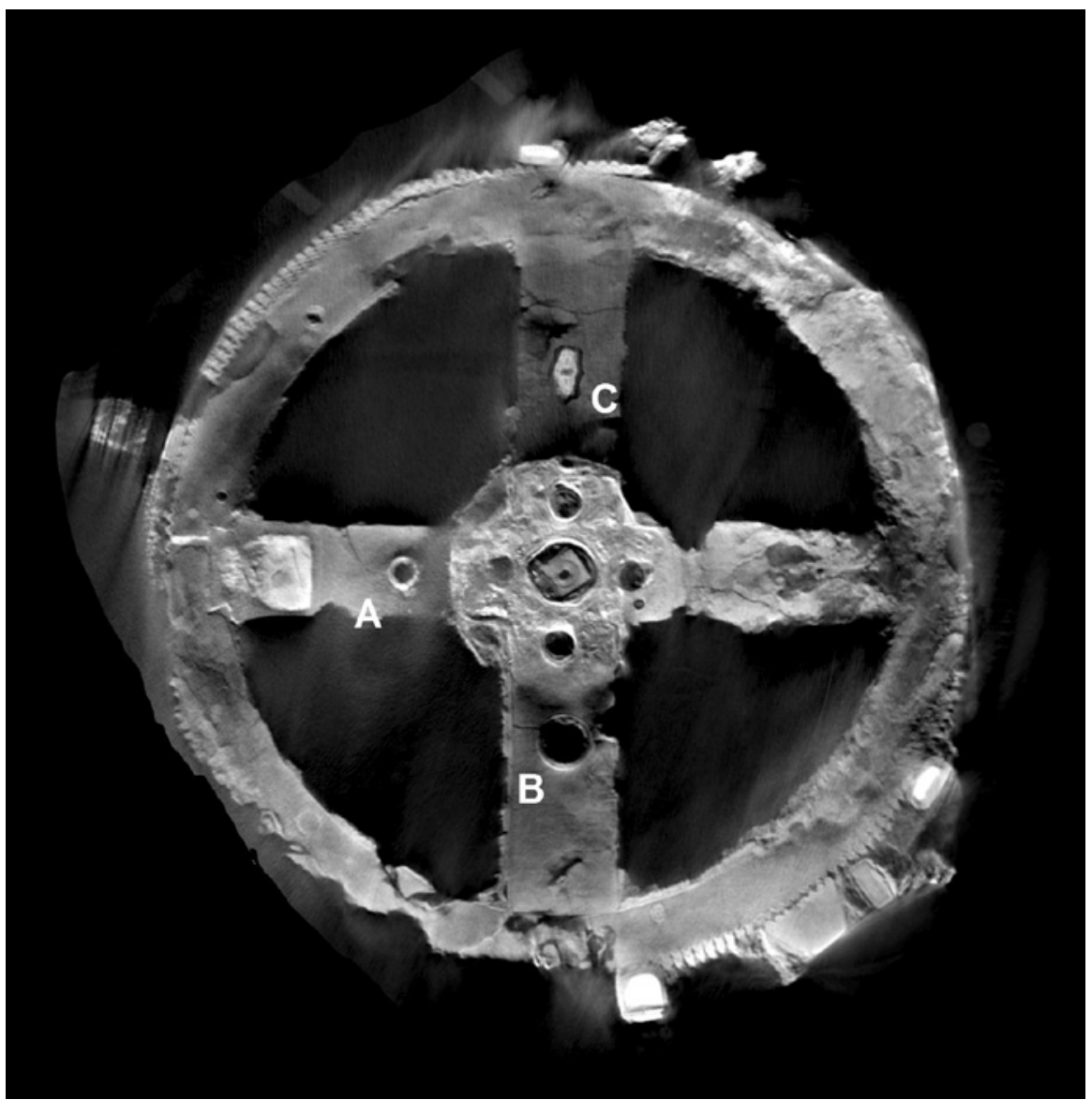

FIG. 8. Composite x-ray of the main solar gear b1. Copyright of the Antikythera Mechanism Research Project.

of teeth on wheel y that have been engaged is given by

$$
\begin{gathered}
m=y \phi / 360 \\
=(\theta / 360)(u y / x) .
\end{gathered}
$$

The total space rotation of wheel $\mathrm{z}$ is $\psi$ (assumed clockwise). The number of teeth engaged on $\mathrm{z}$ is $z(\theta-\psi) / 360$, and this must be equal to $m$. So we obtain

$$
\psi=\theta(1-u y / x z) \text {. }
$$

Thus, since $\theta$ turns at the frequency $\omega_{\odot}$ of the main solar gear,

$$
\omega_{\mathrm{z}}=\omega_{\odot}(1-u y / x z)
$$

If the tooth numbers are chosen appropriately, $\omega_{\mathrm{z}}$ can be made equal to $\omega_{\mathrm{si}}$. 

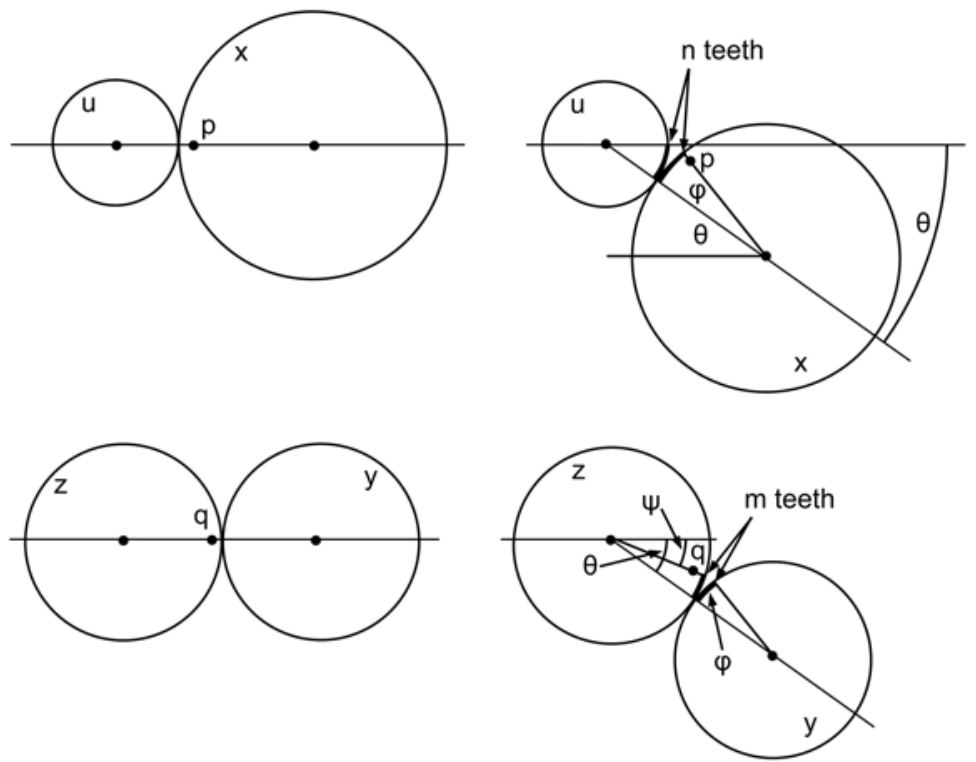

FIG. 9. Obtaining the mean sidereal motion of an outer planet from the mean motion of the Sun.

Here is an alternative, and shorter, argument. Let $\mathrm{u}$ and $\mathrm{z}$ rotate independently about a common axis, and let $\mathrm{x}$ and $\mathrm{y}$ rotate at the same rate as one another about their common axis. Suppose $\mathrm{x}$ and y rotate clockwise by an angle $\phi$. Then $\mathrm{x}$ drives $\mathrm{u}$ in counterclockwise rotation by an angle $-u \phi / x=-\theta$ say. Similarly y drives $\mathrm{z}$ in counterclockwise rotation by an angle $-y \phi / z=-\Omega$ say. To get to a final configuration where $\mathrm{u}$ has remained fixed, we rotate the entire assembly as a rigid body by an angle $\theta$. This returns $\mathrm{u}$ to its initial orientation. The final orientation of $\mathrm{z}$ is $-\Omega$ $+\theta$, which is $\psi=-y \phi / \mathrm{z}+u \phi / x$. Since $\phi=x \theta / u$, we get $\psi=(1-u y / x z) \theta$, as before.

Note that $\omega_{\mathrm{z}}$ can be of either sign, depending on the tooth numbers. We want $\omega_{\mathrm{z}}$ positive, i.e., with $\mathrm{z}$ revolving in the same direction as the Sun, and equal to $\omega_{\text {si, }}$, the sidereal frequency of the superior planet. So we obtain

$$
u y / x z=1-\omega_{\mathrm{si}} / \omega_{\odot},
$$

or, since for a superior planet $\omega_{\mathrm{si}}+\omega_{\mathrm{a}}=\omega_{\odot}$,

$$
u y / x z=\omega_{\mathrm{a}} / \omega_{\odot} .
$$

An appropriate choice of gears can be determined from the relation between the year and the anomalistic period of the planet, which can be drawn from a Babylonian period relation. It is easiest to work with the Babylonian goal-year periods (rather than the ACT periods), as they involve generally small integers. ${ }^{20}$ For example, one period relation attested for Mars is

79 years $=42$ sidereal periods $=37$ anomalistic (or synodic) periods [Mars]. 
So, to have gear z turn at the sidereal frequency of Mars, we need $(u / x) \times(y / z)=37 / 79$. A gear scheme that will work nicely is shown in Figure 7A or 7B. For here we have

$$
(u / x)(y / z)=(37 / 79) \times(58 / 58)=37 / 79,
$$

as required, while we also have $u+x=y+z=116$, which means the gears will mesh as required. Here, for simplicity, we have taken all the gears in the four-gear train to be of the same module. But wheels y and $\mathrm{z}$ could be of any convenient tooth number, or of any convenient module, as long as they have the same tooth count and module as one another. As we shall see, we will need this flexibility later on.

Similarly, using the Babylonian goal-year periods for Jupiter and Saturn,

71 years $=6$ sidereal periods $=65$ anomalistic periods [Jupiter]

59 years $=2$ sidereal periods $=57$ anomalistic periods [Saturn], we can construct the solutions

for Jupiter: $\quad u, x, y, z=65,71,68,68$, so $(u / x)(y / z)=65 / 71$, where $u+x=136$ and $y+z=136$;

and for Saturn: $u, x, y, z=57,59,58,58$, so $(u / x)(y / z)=57 / 59$, where $u+x=116$ and $\mathrm{y}+\mathrm{z}=116$.

We now have all the elements we need to construct a workable solution for the superior planets: the big carrier gear b1, turning at the rate of the Sun; a demonstration that the point-and-slot mechanism can mimic the Apollonian theory in angle; and three gears turning at the sidereal rates of Mars, Jupiter, and Saturn.

\section{Putting It All Together}

Figure 10 shows an obvious concatenation of the results above: we append the pinand-slot construction to the output of the mean sidereal motion of Mars. Gears $\mathrm{u}, \mathrm{x}$, $\mathrm{y}$, and $\mathrm{z}$ result in $\mathrm{z}$ rotating with respect to absolute space at $\omega_{\text {si }}$, as shown above. (The black diamonds on the axis remind us that gears $\mathrm{x}$ and $\mathrm{y}$ are fused together.) Then gears $\mathrm{z}, \mathrm{a}, \mathrm{b}$, and $\mathrm{c}$, with the pin-and-slot mechanism linking a and $\mathrm{b}$, produce the modulated motion, with synodic oscillation superimposed. The Mars axis, connected to $\mathrm{c}$, has the correct mean motion around the zodiac, and an anomalistic motion of the correct period. A Mars pointer could be connected to this axis. It is noteworthy that all the gear axes are either concentric with the main solar axis, or else ride around on the main solar gear. The size of the retrogradations may be adjusted by making use of the parameters of the pin and slot: the key ratio is the eccentricity of wheel $\mathrm{b}$ to the distance of the pin from the centre of wheel a. This solution requires seven gears. Similar solutions can be formed for Jupiter and Saturn.

Glancing at the reconstruction in Figure 10, one might be struck by its wastefulness: look at all those 58-tooth wheels. And note how the four-gear train made by the mechanism for the sidereal motion seems to be echoed by the four-gear train for the anomaly. Wouldn't it be possible to do the whole thing (mean sidereal motion 


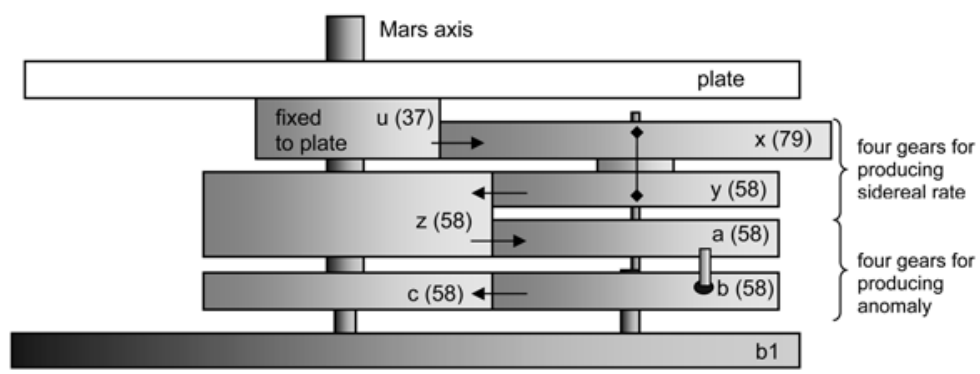

FIG. 10. Complete solution for Mars, embodying the correct sidereal period and the correct anomalistic period. Seven gears in all are used.

plus anomaly), using only four gears? In Figure 10, we see that $\mathrm{y}$ is driving $\mathrm{z}$, and that $\mathrm{a}$ is concentric with $\mathrm{y}$ and is driven by $\mathrm{z}$. Thus $\mathrm{y}$ and a rotate at the same rate (they are both meshed with the same gear and are on the same axis). So y and a can be fused into one, as in Figure 11. But, now we can take another step and eliminate $\mathrm{z}$ in Figure 11, for $\mathrm{y} / \mathrm{a}$ is moved directly by $\mathrm{x}$, and $\mathrm{z}$ is therefore useless. But then the tooth count of $y / a$ becomes irrelevant, and y/a may be considered simply to be a part of $\mathrm{x}$. Thus we arrive at Figure 12A, where the $\mathrm{z}$ of Figure 12A is the $\mathrm{c}$ of Figure 11, and the $\mathrm{y}$ of Figure 12A is the $\mathrm{b}$ of Figure 11. (An alternative, but equivalent, solution is shown in Figure 12B, in which the stationary gear is mounted to the boss below b1 instead of to the plate above it.)

We offer now an analytical demonstration, showing that the four-gear solutions of Figure 12A or Figure 12B indeed have all the right properties — the right sidereal period and the right anomalistic period. Equation 2 requires

$$
\omega_{\mathrm{si}}=\omega_{\odot}(1-u y / x z) \text {. }
$$

Thus the sidereal period will be fine as long as we do not disturb the ratio $(u y) /(x z)$. Now, Equation 3 requires

$$
\omega_{\mathrm{a}}=\omega_{\odot}(u y / x z) .
$$

But, in the right sides of Figure 9, we need $\phi$ to be equal to $\alpha$, the increase in epicyclic anomaly. This is because $\phi$ indicates how far point $p$ has moved around the anomalistic cycle, carrying the pin that is a part of the pin-and-slot mechanism. Imposing this condition on Equation 1 requires

$$
\omega_{\mathrm{a}}=\omega_{\odot}(u / x) .
$$

Comparing the last two equations, we see that we must have $y=z$, which is in fact the way we have drawn Figure 12A (and 12B). With this choice, the four-gear solution of Figure 12A (or 12B) builds in not only the correct sidereal motion, but also a pin-and-slot device producing the right anomalistic motion. 


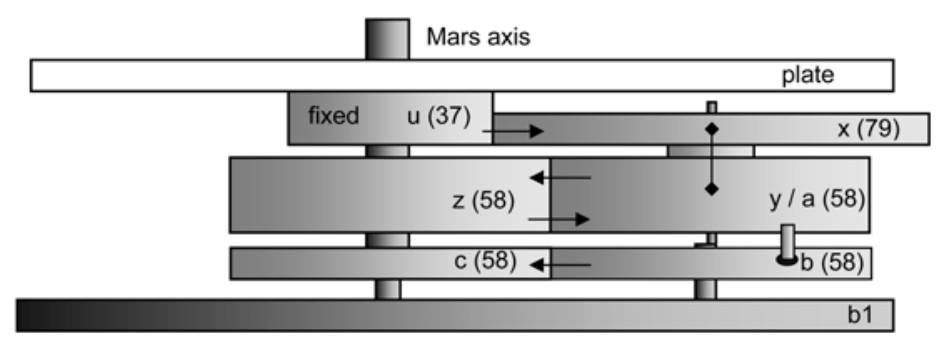

FIG. 11. A step on the way to a simpler solution.

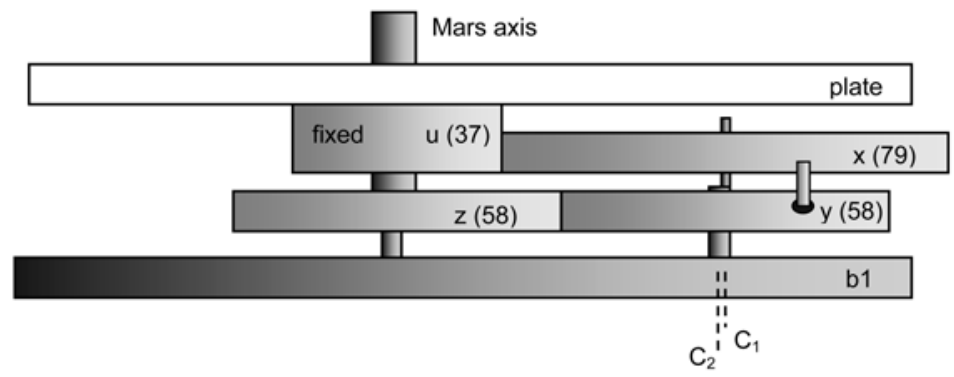

FIG. 12A. Four-gear solution equivalent to Fig. 10. Stationary gear mounted to a plate nearer the front of mechanism.

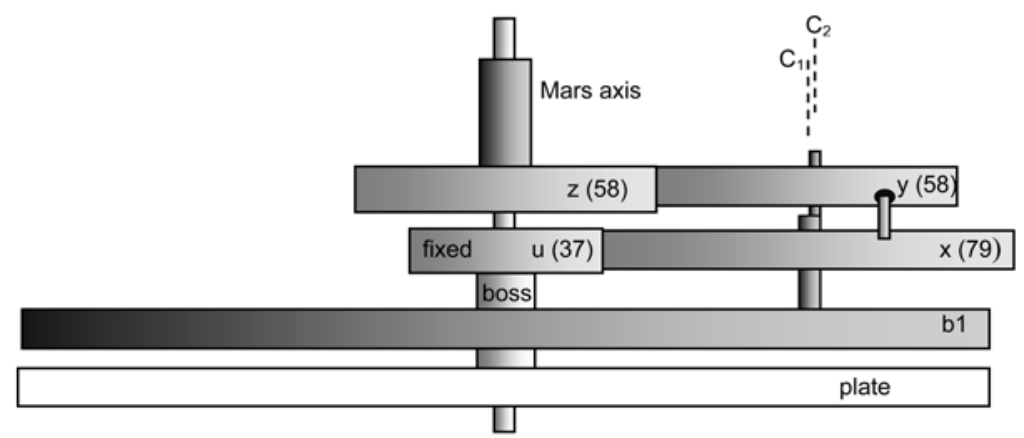

FIG. 12B. Alternative four-gear solution, with stationary gear mounted on a boss fixed to the plate behind b1.

\section{A Proposal}

In Figure 8, the holes at $\mathrm{A}$ and $\mathrm{B}$ and the lug at $\mathrm{C}$ are placed more or less at equal distances from the centre of the wheel. The X-ray shown is a composite: one X-ray slice shows most of the surface of the wheel, including holes A and B; but a portion of a second x-ray slice taken several millimetres higher has been added to show the lug. This lug stands about $6 \mathrm{~mm}$ high. We imagine that apparatus was dropped over it and secured to it. Perhaps another lug was originally fastened at A. ${ }^{21}$ 
The construction described above, using pin-and-slot mechanisms for the outer planets, lends itself well to this geometry. See Figure 13. The solutions for each of the superior planets may be mounted on b1, taking advantage of what appear to be mounting holes on two of the spokes, and the lug on another spoke, using gear modules (millimetres of gear diameter per tooth) that are all in the vicinity of the gear modules typical of the Antikythera mechanism (on the order of $0.5 \mathrm{~mm}$ per tooth). The lug perhaps served to raise the gears vertically. Indeed, it would be necessary for the $y$ and $\mathrm{z}$ gears of the three planets to be placed at different heights. In Figure 13 , the nomenclature "xj 71" means "gear $x$ for Jupiter with 71 teeth". The gears u, $\mathrm{x}, \mathrm{y}$ and $\mathrm{z}$ for each planet are named as in Figure 12A. For each planet, a stepped stud is fastened to wheel b1. The wheel carrying the pin rotates about centre $\mathrm{C}_{1}$ and the wheel with the slot rotates about centre $\mathrm{C}_{2}$. The centre of the larger-diameter part of the stud is marked + and the smaller-diameter part of the stud is indicated $\oplus$.

The simplification that we can simultaneously require $u+x=y+z$ and maintain a constant gear module throughout the four-gear train, which was fine for the case of a zero-eccentricity diagram such as Figure 6, cannot be maintained once eccentricity is introduced. The construction of Figure 13 is based on always putting the gear modules near to $0.5 \mathrm{~mm}$ of diameter per tooth (where the diameter is the pitch diameter), in the range typical of the Antikythera mechanism. But the module for a given planet's $\mathrm{y}$ and $\mathrm{z}$ is not always exactly the same as the module for $\mathrm{u}$ and $\mathrm{x}$.

Mars provides a minor extra challenge because of the large eccentricity $\mathrm{C}_{1} \mathrm{C}_{2}$ (corresponding to a large epicycle in the Apollonian theory). The slot in wheel $\mathrm{y}$ is directed toward $\mathrm{C}_{2}$; but because $b / r>0.5$, the slot would need to be so long that it would overlap the part of the stud that serves as the $\mathrm{C}_{2}$ axis, if we use the arrangement shown in Figure 12A. There are any number of ways to deal with this. An easy solution is simply to reverse the direction of the stepped stud shown in Figure 12A, so that the small end of the stud goes into b1. A metal strap would go over the back of the gears and securely hold the wide end of the stud above wheel x. (It should be noted that 'above' and 'below' are terms that apply only to the diagrams: in the normal operation of the Antikythera mechanism, all the axles shown in Figure 12A would be horizontal.) A strap might be in any case a requirement for stability of the gear trains; and such a strap was a part of the extant lunar anomaly mechanism. This is the arrangement on which Figure 13 is based. An alternative choice would be a different arrangement of the gears, so that wheel $\mathrm{x}$ could be closer to b1 than is wheel $\mathrm{y}$; this would make the $\mathrm{C}_{2}$ part of the stud narrower than the $\mathrm{C}_{1}$ part of the stud (as in Figure 12B).

None of the details of Figure 13 should be given much weight — there is simply too little material evidence and there would be many different ways of mounting the gears. We intend the figure only as an illustration of the principle that a pin-and-slot solution for the superior planets is workable. 


\section{Calibration Issues for the Superior Planets}

In Apollonian planetary theory (Figure 5), let $R_{\mathrm{e}}$ denote the radius of the epicycle and $R_{\mathrm{d}}$ the radius of the deferent circle. In the corresponding pin-and-slot representation (Figure 6), let $r\left(=\mathrm{C}_{1} \mathrm{D}\right)$ represent the distance of the pin from $\mathrm{C}_{1}$; and let $b$ $\left(=\mathrm{C}_{1} \mathrm{C}_{2}\right)$ represent the off-centredness of circle $\mathrm{k} 2$ with respect to circle $\mathrm{k} 1$.Then the pin-and-slot mechanism will give behaviour corresponding to the deferent-andepicycle theory if

$$
b / r=R_{\mathrm{e}} / R_{\mathrm{d}} .
$$

One might worry that the fact that gears $y$ and $\mathrm{z}$ in Figure 12A have different numbers

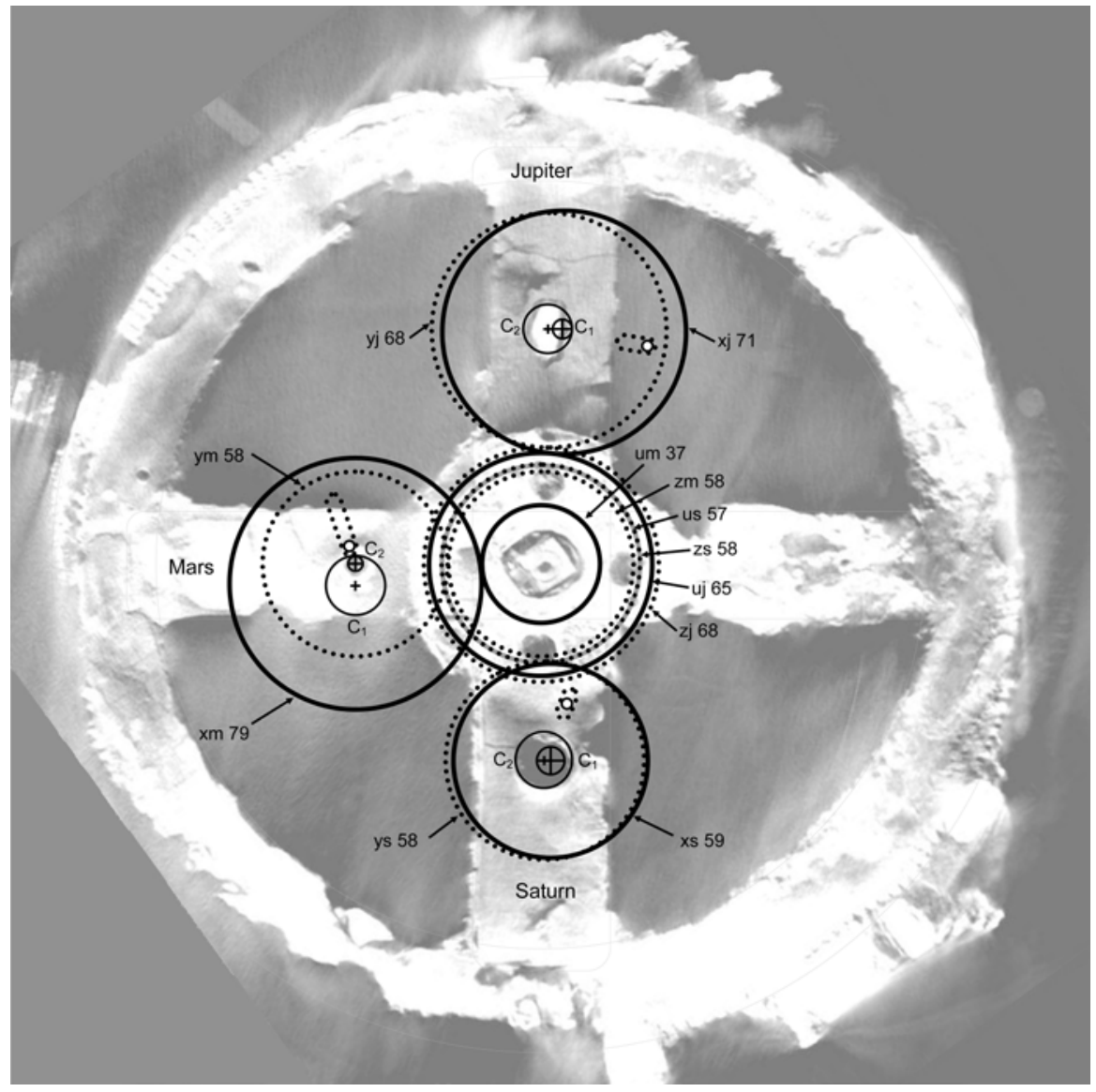

Fig. 13. A possible kinematic solution for the superior planets, based on the pin-and-slot construction, superimposed on the main solar wheel b1. Underlying x-ray copyright of the Antikythera Mechanism Research Project. 
of teeth than gear $\mathrm{x}$ might require some sort of adjustment to this relationship. But no adjustment is required. In Figure 12A, the angular oscillation (with respect to the mean motion) of gear $\mathrm{y}$ is determined by the ratio $\mathrm{b} / \mathrm{r}$, and the tooth count of $\mathrm{y}$ doesn't enter into it. But then the angular oscillation of $\mathrm{z}$ will be the same as that of $\mathrm{y}$, since $\mathrm{z}$ and $\mathrm{y}$ do have the same number of teeth.

In Figure 13, we have for the sake of simplicity shown the $\mathrm{C}_{1}-\mathrm{C}_{2}$ lines for Jupiter and Saturn as parallel to one another. This might facilitate setting initial conditions, but it is not a necessary mechanical arrangement, which we emphasize by setting the $\mathrm{C}_{1}-\mathrm{C}_{2}$ direction for Mars at right angles to those for the other planets. The $\mathrm{C}_{1}-\mathrm{C}_{2}$ directions can be chosen at will, and separately for each planet. Suppose that the actual planet is in the middle of retrograde motion. Then we want to set the gears for its pin-and-slot mechanism so that the pin is closest to $\mathrm{C}_{2}$. This guarantees that the phasing of the pin-and-slot mechanism matches the retrograde cycle of the actual planet. Then, the pointer that indicates the planet's longitude should be set so that it points diametrically opposite the Sun.

\section{Inferior Planets}

The fourth spoke is free for the inferior planets, which can be accommodated in a more ordinary way, using a simple epicycle construction, as shown in Figure 14. A construction resembling Figure 14 was described by Edmunds and Morgan. ${ }^{22}$ And Wright used a similar construction for the inner planets, with, however, compound gear trains rather than simple gear pairs. ${ }^{23}$ As the sidereal frequency of both Mercury and Venus is exactly $\omega_{\odot}$, both planets' apparatus may be mounted directly on the fourth spoke of b1. If the fixed wheel has $u$ teeth and the epicycle wheel has $x$, then the anomalistic frequency is given by $\omega_{\mathrm{a}}=\omega_{\odot}(u / x)$. If $R_{\mathrm{e}}$ and $R_{\mathrm{d}}$ represent the radii of the epicycle and of the deferent in standard theory, we should then require DP/ $\mathrm{CD}=R_{\mathrm{e}} / R_{\mathrm{d}}$.

\section{Trial by Fire}

We actually built a Mars model in metal, based on the construction of Figure 12A and Figure 13, using the pin-and-slot mechanism to model the inequality. We can confirm that it works smoothly, even for a planet with such a large Apollonian epicycle. One point that modellers should be aware of is that the roles of the pin and the slot cannot be reversed. For Mars, having the slot lead the pin (as in Figure 4, right) would result in a model in which retrograde motion does not actually occur. This results from the two different mathematical forms for the equation $q$ in these two cases, combined with the fact that for Mars $\omega_{\text {si }}$ is slightly larger than $\omega_{\mathrm{a}}$. For a planet such as Saturn, for which $\omega_{\mathrm{a}} \gg \omega_{\text {si }}$ and the epicycle is small, one could reverse the roles of the pin and slot without dire consequences; the pin and slot would no longer perfectly mimic the epicycle model in angle, but the results would still be tolerably good.

With the correct version of the model (i.e., with the pin leading the slot), the 


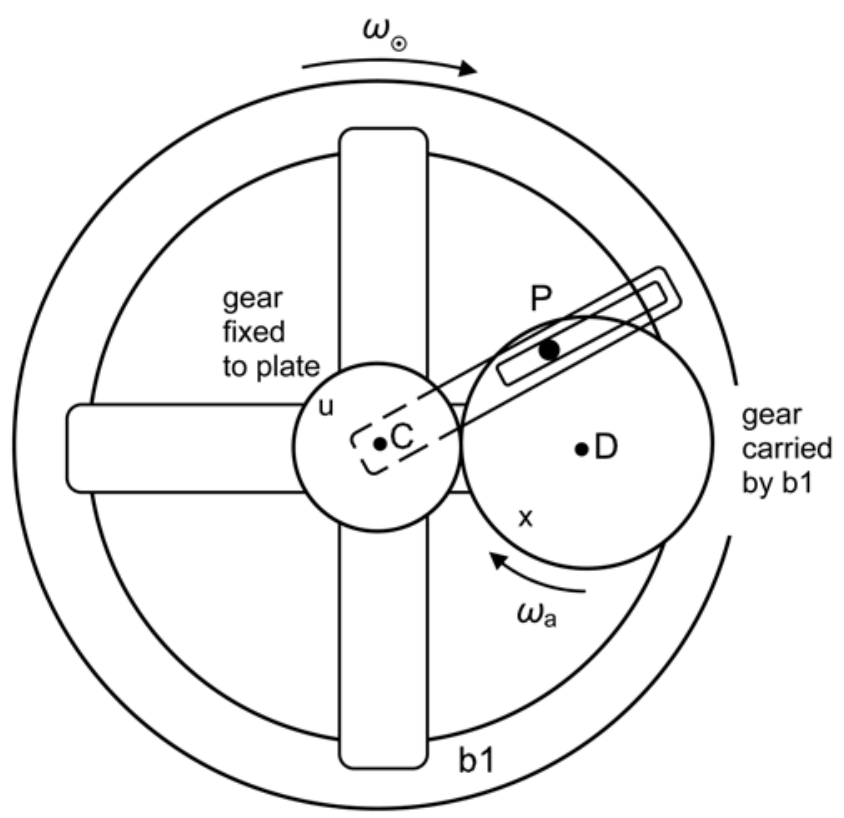

FIG. 14. A possible epicyclic construction for an inner planet.

pin-and-slot mechanism matches the Apollonian theory in angle. But Apollonian planetary theory itself gives but a poor approximation to the actual motion of Mars. ${ }^{24}$ The combination of large epicycle and a large eccentricity required something more complex than the simple theory of Apollonios for Greek planetary theory to yield a satisfactory representation of the motion of Mars.

\section{Concluding Remarks}

Unless new material is discovered, it is conceivable that we will never know for sure just how the planets were represented on the Antikythera mechanism. But the solution for the superior planets described here has great attraction. It uses for these planets' inequality with respect to the Sun precisely the mechanism that we know was used for producing the lunar inequality with respect to the zodiac. And, as we have shown, this solution is an exact representation in angle of Apollonian epicycle theory. Moreover, this solution requires but four gears for each of the superior planets, it explains why gear b1 needed to be large, and it can be fitted well to the construction holes that remain on b1. Of course, one could object that a complete model would require many concentric axles - and so one's receptiveness to the proposal may in part depend on what sort of mechanical engineering one considers likely around the second century B.C. ${ }^{25}$ The chief disadvantage of the present proposal is that it offers no explanation of the tooth count of b1. It also does not explain the need for the large crossed-out 
regions of $b 1$; perhaps these were only access ports. Or perhaps they were connected with a display of the four seasons, as we have suggested elsewhere. ${ }^{26}$ In any case, we hope that this paper has offered some insights into the richness of the pin-and-slot mechanism and that it will serve to focus new attention on the issues raised by the intersection of ancient Greek theoretical astronomy and ancient Greek mechanics.

\section{ACKNOWLEDGEMENT}

We are grateful to Mike Edmunds and Tony Freeth of the Antikythera Mechanism Research Project for their generosity in making data available to us.

\section{REFERENCES}

1. T. Freeth, Y. Bitsakis, X. Moussas, J. H. Seiradakis, A. Tselikas, H. Mangou, M. Zafeiropolou, R. Hadland, D. Bate, A. Ramsey, M. Allen, A. Crawley, P. Hockley, T. Malzbender, D. Gelb, W. Ambrisco, and M. G. Edmunds, "Decoding the ancient Greek astronomical calculator known as the Antikythera mechanism", Nature, cdxliv (2006), 587-91. There is substantial "Supplementary information" linked to the online version of the paper at www.nature.com/nature.

2. Derek de Solla Price drew attention to this slot, but conjectured that it might be the result of an attempt to repair a broken gear. Gears from the Greeks: The Antikythera mechanism - A calendar computer from ca. 80 B.C. (Transactions of the American Philosophical Society, n.s., 1xiv/7 (1974)), 35. M. T. Wright described the pin-and-slot device and its workings and observed that it would suitable for modelling an anomaly, in "Epicyclic gearing and the Antikythera mechanism, part 2", Antiquarian horology, xxix/1 (September 2005), 51-63.

3. Freeth et al., "Decoding" (ref. 1), Supplementary information, 25-7.

4. J. Evans, C. C. Carman and A. S. Thorndike, "Solar anomaly and planetary displays in the Antikythera mechanism," Journal for the history of astronomy, xli (2010), 1-39. The evidence is very simple and comes from matching degree marks on the zodiac scale with day marks on the Egyptian calendar scale. Over a preserved stretch of $69^{\circ}$ of the calendar scale, an apparent equation of centre rises steadily to a $2.1^{\circ}$ effect, of the right sign and amplitude to represent the Sun's inequality. We shall shortly publish a second study of the zodiac and Egyptian calendar scales, examining this issue in greater detail.

5. Price, Gears from the Greeks (ref. 2), 21. M. T. Wright, "A planetarium display for the Antikythera mechanism", Horological journal, cxliv (2002), 169-73 and 193; and "The Antikythera mechanism: A new gearing scheme", Bulletin of the Scientific Instrument Society, 1xxxv, issue of June 2005, 2-7. Freeth et al., "Decoding" (ref. 1), 590.

6. Freeth et al., "Decoding" (ref. 1), Supplementary information, 8-9, with translation on pp. 10-14.

7. Wright, "A planetarium display" (ref. 5). A film of Wright's reconstruction in motion can be seen at www.youtube.com/watch? $\mathrm{v}=\mathrm{ZrfMFhrgOFc}$ This film was made in 2008 by the science writer Jo Marchant, who was then working for New scientist.

8. Evans, Carman and Thorndike, "Solar anomaly" (ref. 4).

9. Theon of Smyrna, Mathematical knowledge useful for reading Plato, iii, 26; J. Dupuis, transl., Théon de Smyrne, philosophe platonicien: Exposition des connaissances mathématiques utiles pour la lecture de Platon (Paris, 1892), 269.

10. We follow the gear names used by Freeth et al. in "Decoding" (ref. 1), which in some cases represent departures from Price's nomenclature in Gears from the Greeks (ref. 2).

11. The output is by means of a central shaft attached to e6; and this shaft runs inside the hollow pipe to which e5 is attached.

12. Ptolemy's proof of the equivalence for the Sun is in Almagest iii, 3, and for the Moon at iv, 5. G. J. Toomer, Ptolemy's Almagest (London, 1985), 141-53 and 180-90. Ptolemy's remarks about 
Apollonios are in Almagest xii, 1 (Toomer, p. 555). The classic argument that Apollonios understood the equivalence of epicycles and eccentrics is Otto Neugebauer, "The equivalence of eccentric and epicycle motion according to Apollonios", Scripta mathematica, xxiv (1959), 5-21, recapitulated in A history of ancient mathematical astronomy (Berlin and New York, 1975), 149-50, 267-70. But scholars have sometimes questioned the correctness of attributing this understanding to Apollonios. See, most recently, Bernard R. Goldstein, "Apollonios of Perga's contributions to astronomy reconsidered", Physis, xlvi (2009), 1-14. For Theon of Smyrna's proofs see Dupuis, Théon de Smyrne (ref. 9), 271-9.

13. Alexander Jones, Astronomical papyri from Oxyrhynchus (Memoirs of the American Philosophical Society, ccxxxiii; Philadelphia, 1999).

14. See, e.g., B. R. Goldstein, "Descriptions of astronomical instruments in Hebrew”, in D. A. King and G. Saliba (eds), Essays in honor of E. S. Kennedy (Annals of the New York Academy of Sciences, d (1987)), 105-41, pp. $106 \mathrm{ff}$.

15. The expressions for $\sin q$ are the same pair that we get if, in the ordinary eccentric-circle theory, we express $q$ first as a function of the mean anomaly and then as a function of the true anomaly. See James Evans, The history and practice of ancient astronomy (New York, 1998), 233-4.

16. Freeth et al., "Decoding" (ref. 1), Supplementary information, 24. We have modified this quotation by multiplying all the frequencies by a minus sign, in order to make the discussion more easily comparable with our diagrams.

17. This is a distinction in language used by Ptolemy himself (that one sort of anomaly is related to the Sun, and is one related to position in the zodiac), e.g., in Almagest ix, 2; Toomer, Ptolemy's Almagest (ref. 12), 420.

18. See, for example, Evans, History and practice (ref. 15), 338.

19. We thank Michael Wright for this suggestion (private communication), as well as for several other comments that helped improve the paper.

20. Listed in Evans, History and practice (ref. 15), 314. The term 'goal-year texts' was introduced by Abraham Sachs, "A classification of the Babylonian astronomical tablets of the Seleucid period", Journal of cuneiform studies, ii (1970), 271-90.

21. Price, Gears from the Greeks (ref. 2), 28, speculated that there might have been other lugs fastened to b1.

22. Mike Edmunds and Philip Morgan, "The Antikythera mechanism: Still a mystery of Greek astronomy?", Astronomy \& geophysics, xli, issue of December 2000, 6.10-6.17.

23. Wright, "A planetarium display" (ref. 5).

24. See Evans, History and practice (ref. 15), 341.

25. In arguing that multiple concentric pipes would not be a problem, Wright makes an analogy to surviving fragments of the musical wind instrument called the aulos, in which rotatable sleeves of bronze tube are often seen fitted over a structural tube. M. T. Wright, "In the steps of the master mechanic", in Ancient Greece and the modern world (Patras, 2003), 86-97.

26. Evans, Carman and Thorndike, "Solar anomaly" (ref. 4), 32-3. 